\title{
Revisión Sistemática: ¿Es recomendable el rastreo de la depresión en adultos?
}

Screening for Depression in Adults: A Summary of the Evidence for the U.S. Preventive Services Task Force. Pignone M, Gaynes B, Rushton J, et al. Ann Intern Med 2002; 136:765-776

\section{Objetivo}

Establecer si el rastreo sistemático de depresión en centros de atención ambulatoria de adultos mejora la detección, el tratamiento y la evolución de los pacientes deprimidos.

\section{Fuente y selección de datos}

Se revisó MEDLINE y Colaboración Cochrane desde 1994 hasta 2001. Se focalizó la busqueda en estudios controlados aleatorizados realizados en atención primaria que compararan el efecto del rastreo rutinario de depresión versus el cuidado usual evaluando la frecuencia de detección, indicación de tratamiento y el impacto en la evolución clínica.

\section{Resultados principales}

Se seleccionaron 14 estudios. Las variables analizadas fueron: diferencias en las tasas de detección de depresión, diferencias en la proporción de pacientes con depresión que iniciaron tratamiento o fueron derivados para ello, y cambios en la evolución clínica. Los estudios difirieron en el grado de intervención. Todos utilizaron inicialmente algún cuestionario para la detección de depresión. Los pacientes deprimidos así detectados, fueron aleatorizados de modo tal que el médico a cargo conocía el diagnostico en aproximadamente la mitad de los casos (grupo intervención) y desconocía el diagnóstico en la otra mitad (grupo control o de cuidado usual). A esta intervención se la denominó "devolución de resultados de rastreo". En 7 de 14 estudios se evaluó el efecto de esta intervención sobre la tasa de detección de depresión, la cual se incrementó entre un 10 y un $47 \%$ a favor del grupo intervención. Cuando se evaluó el efecto de la "devolución" sobre la proporción de inicio de tratamiento, los resultados fueron mixtos, observándose un efecto favorable en forma significativa en 4 de los 7 estudios que aportaban este dato. Lo mismo sucedió al evaluar este efecto sobre la evolución clínica, donde 5 de 9 trabajos mostraron resultados favorables en forma significativa.

Algunos estudios realizaron además otra intervención que consistía en dar consejos generales o específicos acerca del tratamiento. Otros ofrecieron al médico tratante recursos sistematizados para aumentar la calidad del tratamiento y seguimiento posterior.

Debido a que varios de los estudios no alcanzaban el poder suficiente para detectar cambios en los resultados clínicos se realizó un meta-análisis que mostró un Riesgo Relativo de 0.87 (IC95\% 0.79 a 0.95 ) de permanecer deprimido luego de la intervención y una Reducción de Riesgo Absoluto de 9\% (IC95\% 4 a 14\%) a favor del grupo en el que se realizó la intervención. Un meta-análisis ulterior excluyendo un estudio, mostró un riesgo relativo de 0.90 (IC95\% 0.82 a 0.98), una Reducción de Riesgo Absoluto de $7 \%$ (IC95\% 3 a $11 \%$ ) y con una menor heterogeneidad* entre los estudios $(P=0.16)$.

\section{Conclusión}

El rastreo de depresión en centros de atención primaria mejoraría la frecuencia de detección, de tratamiento y la evolución de los pacientes, especialmente si es acompañado de medidas de soporte y tratamiento.

\section{Comentario}

Este estudio es una revisión sistemática de la Fuerza de Tareas en Prevención de los EE.UU (USPSTF) con el propósito de analizar la evidencia acerca de la utilidad de rastrear sistemáticamente depresión en atención primaria. Si el rsatreo puede aumentar la proporción de pacientes que logran una remisión en un $9 \%$ a los seis meses, cerca de 11 pacientes deprimidos deberían identificarse para lograr una remisión adicional. Si tenemos en cuenta que la prevalencia de depresión que responde al tratamiento es del $10 \%$, se deben rastrear 110 pacientes para producir una remisión adicional a los seis meses (Número Necesario a Rastrear $^{*}$.

En esta revisión no están exhaustivamente explicados los criterios de selección de los artículos, los cuales por otro lado presentan diferencias sustanciales en el tamaño muestral, en los instrumentos utilizados para detectar depresión y en las intervenciones realizadas. Esto explica la disparidad de los resultados.

Estas objeciones no son suficientes para desestimar los resultados observados y por ello la USPSTF ha establecido como recomendación de grado $\mathrm{B}^{\star}$ el rastreo de depresión en centros de atención ambulatoria que cuenten con servicios de atención para los casos diagnosticados.

En este trabajo, el diagnóstico de depresión lo realizó un profesional diferente del médico tratante, lo cual no ocurre en la consulta habitual donde la detección y la decisión del tratamiento son en general responsabilidad del médico general. Debido esto es dudoso que el beneficio observado en esta revisión sistemática sea el alcanzado en la práctica clínica habitual.

Diferentes investigaciones han demostrado la alta prevalencia de depresión en centros de atención primaria. En general los pacientes deprimidos consultan por síntomas sómaticos y no por alteraciones del estado de ánimo'; por lo tanto es importante que los médicos de atención primaria estén sensibilizados y adquieran entrenamiento en la detección de las distintas formas de presentación de esta enfermedad.

Considerando que se estima que la depresión será la segunda causa de morbilidad en los próximos veinte años, es necesario mejorar las estrategias diagnósticas en los consultorios de atención ambulatoria.

Conclusiones del comentador: La fuerza de tareas americana recomienda rastrear depresión en centros ambulatorios que cuanten con servicios de atención para la misma. A pesar de esto, debido a la particular situación socioeconómica que atraviesa nuestro país y la alta prevalencia de depresión, es importante realizar el rastreo si uno luego puede ayudar a los pacientes que la padezcan. 